\title{
Neural substrates related to auditory working memory comparisons in dyslexia: An fMRI study
}

\author{
TIM CONWAY, ${ }^{1,2,3,4}$ KENNETH M. HEILMAN,,${ }^{1,2,4,5}$ KAUNDINYA GOPINATH, ${ }^{6}$ \\ KYUNG PECK, ${ }^{7}$ RUSSELL BAUER, ${ }^{2,4}$ RICHARD W. BRIGGS, ${ }^{6}$ JOSEPH K. TORGESEN, ${ }^{8}$ \\ AND BRUCE CROSSON ${ }^{1,2,4}$ \\ ${ }^{1}$ Department of Veterans Affairs Rehabilitation Research and Development Brain Rehabilitation Research Center \\ of Excellence at the Malcom Randall Veterans Administration Medical Center, Gainesville, Florida \\ ${ }^{2}$ Department of Clinical and Health Psychology, University of Florida, Gainesville, Florida \\ ${ }^{3}$ Department of Pediatrics, University of Florida, Gainesville, Florida \\ ${ }^{4}$ McKnight Brain Institute, University of Florida, Gainesville, Florida \\ ${ }^{5}$ Department of Neurology, University of Florida, Gainesville, Florida \\ ${ }^{6}$ Department of Radiology, University of Texas Southwestern Medical School, Dallas, Texas \\ ${ }^{7}$ Department of Medical Physics and Radiology, Memorial Sloan-Kettering Cancer Center, New York \\ ${ }^{8}$ Department of Psychology and Florida Center for Reading Research, Florida State University, Tallahassee, Florida
}

(Received July 31, 2007; Final Revision April 3, 2008; Accepted April 4, 2008)

\begin{abstract}
Adult readers with developmental phonological dyslexia exhibit significant difficulty comparing pseudowords and pure tones in auditory working memory (AWM). This suggests deficient AWM skills for adults diagnosed with dyslexia. Despite behavioral differences, it is unknown whether neural substrates of AWM differ between adults diagnosed with dyslexia and normal readers. Prior neuroimaging of adults diagnosed with dyslexia and normal readers, and post-mortem findings of neural structural anomalies in adults diagnosed with dyslexia support the hypothesis of atypical neural activity in temporoparietal and inferior frontal regions during AWM tasks in adults diagnosed with dyslexia. We used fMRI during two binaural AWM tasks (pseudowords or pure tones comparisons) in adults diagnosed with dyslexia $(n=11)$ and normal readers $(n=11)$. For both AWM tasks, adults diagnosed with dyslexia exhibited greater activity in left posterior superior temporal (BA 22) and inferior parietal regions (BA 40) than normal readers. Comparing neural activity between groups and between stimuli contrasts (pseudowords $v s$. tones), adults diagnosed with dyslexia showed greater primary auditory cortex activity (BA 42; tones $>$ pseudowords) than normal readers. Thus, greater activity in primary auditory, posterior superior temporal, and inferior parietal cortices during linguistic and non-linguistic AWM tasks for adults diagnosed with dyslexia compared to normal readers indicate differences in neural substrates of AWM comparison tasks. (JINS, 2008, 14, 629-639.)
\end{abstract}

Keywords: Reading, Language, Linguistics, Adult, Cognition, Speech

\section{INTRODUCTION}

Developmental dyslexia is a neurobiological disorder characterized by a deficit in the phonological component of language (speech sounds) and poor reading skills. These deficits occur in the relative absence of other cognitive def-

Correspondence and reprint requests to: Tim Conway, Ph.D., Department of Clinical and Health Psychology, University of Florida Health Science Center, Box 100165, Gainesville, FL 32610-0165. E-mail: twc@phhp.ufl.edu icits and neurological disorders (Lyon et al., 2003; Shaywitz et al., 2008). Developmental dyslexia is also associated with deficient memory skills and specific deficits in auditory working memory (AWM) have been reported (Swanson \& Siegel, 2001; Torgesen, 1985). AWM is the active maintenance and manipulation of a finite amount of verbal information for a behavioral purpose (Baddeley, 2003). In adult readers with developmental dyslexia, deficient AWM skills may contribute to their reading and phonological deficits (Berninger et al., 2006). However, AWM deficits in 
children diagnosed with dyslexia are also evident during tasks with complex comparisons of non-linguistic (pure tones) stimuli (Banai \& Ahissar, 2006) and the same may be true for adults diagnosed with dyslexia (Chait et al., 2007). Banai \& Ahissar explored the relationship between behavioral task complexity (identification, discrimination, or AWM) and auditory stimuli complexity (tones, phonemes, or pseudowords) in female adolescents with developmental dyslexia. Overall, behavioral performance differences were most evident only when significant working memory demands (identifying-discriminating a stimuli pair from a set of 3 stimuli) were present for pure tone or pseudoword stimuli. Given the evidence of linguistic and non-linguistic auditory working memory deficits in developmental dyslexia, it has been suggested that impaired auditory working memory skills may be one feature of this disorder (Banai \& Ahissar, 2006; Berninger et al., 2006; Kibby et al., 2004).

Post-mortem and structural MRI studies of adults diagnosed with dyslexia identify specific cortical regions that may contribute to deficits in auditory working memory. Postmortem neuroanatomic investigations of adults with developmental dyslexia report perisylvian regions with aberrant neuronal structures (Galaburda et al., 2006; Galaburda et al., 1985). Recent evidence indicates these neuronal anomalies may be neurodevelopmental in nature, originating in utero (Galaburda et al., 2006; Ramus, 2004). The presence of developmental cortical anomalies in perisylvian language cortices of adults with developmental dyslexia suggests that atypical neural activity may be evident in these regions during neuroimaging investigations of AWM.

Neuroimaging studies provide additional evidence about possible relationships between cortical structure, function, and behavioral abilities relative to AWM. Auditory detection and discrimination tasks that had minimal AWM demands (auditory rhyme, phoneme or syllable detection, and phoneme discrimination) have been explored with neuroimaging. When comparing normal readers to adults diagnosed with dyslexia during such tasks, greater left temporoparietal activity in normal readers has been found (Rumsey et al., 1992). In contrast, adults diagnosed with dyslexia have shown higher brain metabolism in the medial temporal lobe during syllable identification than normal readers (Hagman et al., 1992). For normal readers, neuroimaging studies during auditory phonological tasks with only minimal working memory demands report activity in left fusiform gyrus and Broca's region (Demonet et al., 1992), left superior temporal gyrus and bilateral operculum (Fiez et al., 1995), and Broca's area, occipital cortex, and fusiform gyrus (Zatorre et al., 1992; Zatorre et al., 1996). Reportedly, the frontal activity reflects executive control (Hickok \& Poeppel, 2007; Zatorre et al., 1996), rather than storage or manipulation. Other reports contend that AWM tasks can be separated into monitoring and manipulation demands with respective regions of cortical activity. Mid-dorsolateral prefrontal cortex activity may represent monitoring of manipulated stimuli during AWM tasks, whereas manipulation of stimuli may be represented by posterior parietal cortex activity (Berninger et al., 2006; Champod \& Petrides, 2007) (see (Wager \& Smith, 2003) for a review of neuroanatomic, behavioral, and neuroimaging findings in frontal, temporal (excluding superior temporal), and parietal lobes relative to working memory deficits in children and adults diagnosed with dyslexia). Therefore, neuroimaging studies imply that temporal and parietal regions may demonstrate anomalous activity in adults diagnosed with dyslexia.

It is unknown whether normal readers and adults diagnosed with dyslexia will show differential neural activity during AWM tasks with phonological (linguistic) and pure tone (non-linguistic) stimuli. Prior neuroimaging studies of normal readers report overlapping regions in the superior temporal cortex for linguistic and non-linguistic auditory perception (Binder et al., 2000; Liebenthal et al., 2005). Because differential auditory perceptual skills exist between adults diagnosed with dyslexia and normal readers (Tallal, 1980), differential AWM skills may follow and differences between normal readers and those diagnosed with dyslexia during AWM tasks may be observed in superior temporal regions. Unlike neuroimaging during visual reading tasks, which report decreased activity in left inferior temporoparietal regions for adults diagnosed with dyslexia compared to controls (Shaywitz et al., 2008), no between groups differences in left inferior temporoparietal activity have been reported during AWM tasks for adults diagnosed with dyslexia. Overall, differences in neural activity during minimal load AWM tasks may depend on task demands. However, given the temporal cortex's role in primary auditory processing of linguistic and non-linguistic stimuli, the reported differences in neural activity in this region may be particularly relevant to understanding AWM deficits for linguistic and non-linguistic stimuli in adults diagnosed with dyslexia.

This study used fMRI during linguistic (pseudoword) and non-linguistic (pure tone) AWM tasks to identify patterns of neural activity between adults with and without developmental dyslexia. The study's primary aim was to identify if neural substrates of auditory working memory varied between these groups for one or both types of stimuli, pseudowords and pure tones. If between groups differences in neural activity were found, then a secondary aim was to determine if these differences were related to task accuracy, participants' behavioral strategies, degree of behavioral effort, or other factors.

\section{METHODS}

\section{Participants}

Twenty-four monolingual (native English speaking) men (12 normal readers, 12 readers diagnosed with dyslexia) were matched for age, education, general intelligence (IQ), right-handedness (Edinburgh Handedness Inventory) (Oldfield, 1971), and socio-economic status (SES) (Hollingshead, 1975). Also, no group differences existed for parental 
years of education, state anxiety immediately prior to fMRI, and trait anxiety (Spielberger, 1983). Table 1 lists neuropsychological testing that was administered to all participants. Normal readers had average or above skills on all neuropsychological tests. The mean intellectual ability for the adults diagnosed with dyslexia and normal readers was superior, with standard scores ranging from 97 to 143 and 120 to 140 , respectively. Adults diagnosed with dyslexia reported current difficulty with reading and a childhood history of a learning disability. Ten of the adults diagnosed with dyslexia demonstrated reading skills that were at least 1.5 standard deviations below their IQ (meeting an IQ-achievement discrepancy definition of dyslexia), and the eleventh participant's discrepancy was 1.3 standard deviations. The eleventh participant's pseudoword reading was more than three standard deviations below estimated IQ (consistent with a processing deficit definition of dyslexia). Both of these diagnostic approaches identify developmental dyslexia with similar core deficits in phonological abilities (Fletcher \& Shaywitz, 1994; Stanovich \& Siegel, 1994), which persists into adulthood for the current group (Bruck, 1993).
Overall, adults diagnosed with dyslexia demonstrated moderate to severe deficits in oral reading of real words, pseudowords, paragraph reading accuracy, paragraph reading rate, passage comprehension, phonological awareness (CTOPP phonological subtests (Wagner et al., 1999)), and auditory working memory (LAC) (Lindamood, 1985). On another measure of AWM, Auditory Consonant Trigrams (ACT) (Stuss et al., 1988), adults diagnosed with dyslexia showed significant AWM deficits following no delay and 3, 9, and 18 second delays. However, no differences existed between groups on measures of rapid automatized naming (CTOPP; naming subtests) (Wagner et al., 1999). Thus, relative to reading abilities, the adults diagnosed with dyslexia exhibited phonological reading and phonological processing deficits consistent with a diagnosis of developmental phonological dyslexia. The adults diagnosed with dyslexia did not meet criteria for "double-deficit" dyslexia (Wolf \& Bowers, 1999), which is characterized by impaired rapid automatized naming and impaired phonological reading or processing skills.

One typical reader was withdrawn after MRI revealed a left temporal lobe mass and one participant diagnosed

Table 1. Participant demographics and behavioral assessment results

\begin{tabular}{|c|c|c|c|c|c|}
\hline & \multicolumn{2}{|c|}{$\begin{array}{l}\text { Normal Readers } \\
\quad(n=11)\end{array}$} & \multicolumn{2}{|c|}{$\begin{array}{l}\text { Readers diagnosed } \\
\text { with dyslexia } \\
(n=11)\end{array}$} & \multirow{2}{*}{$\begin{array}{c}\begin{array}{c}\text { Statistical } \\
\text { significance }\end{array} \\
p \text {-value }\end{array}$} \\
\hline & $M$ & $S D$ & $M$ & $S D$ & \\
\hline Age (years-months) & $34-10$ & $9-03$ & $35-02$ & $10-10$ & n.s. \\
\hline Education (years) & 15.2 & 2.4 & 14.7 & 2.4 & n.s. \\
\hline $\mathrm{IQ}^{\mathrm{a}}(\mathrm{WAIS}-\mathrm{III})$ & $128.8^{\mathrm{f}}$ & 7.9 & 121.5 & 12.7 & n.s. \\
\hline Handedness ${ }^{b}$ & 88.8 & 10.2 & 78.8 & 14.1 & n.s. \\
\hline Socio-economic Status ${ }^{c}$ & 47.5 & 7.5 & 43.8 & 6.5 & n.s. \\
\hline Nonword Reading (WRMT-R) & $108.6^{\mathrm{f}}$ & 6.1 & 89 & 9.1 & $p<.001$ \\
\hline Real Word Reading(WRMT-R) & $109.6^{\mathrm{f}}$ & 7.4 & 91.8 & 11 & $p<.001$ \\
\hline Rate (GORT-III) & $13.6^{\mathrm{g}}$ & 1.9 & 8 & 5 & $p<.002$ \\
\hline Accuracy (GORT-III) & $14.9^{\mathrm{g}}$ & 1.1 & 7.2 & 5 & $p<.001$ \\
\hline Passage Score (GORT-III) & $14.7^{\mathrm{g}}$ & 1.6 & 7.4 & 4.8 & $p<.001$ \\
\hline Lindamood Auditory Conceptualization Test (LAC) & $96.6^{\mathrm{d}}$ & 3.6 & 82.7 & 9.7 & $p<.001$ \\
\hline LAC-MAC Level 3-Syllable and Phoneme Changes & $90.0^{\mathrm{e}}$ & 11.3 & 62.9 & 16.5 & $p<.001$ \\
\hline Nonword Repetition (CTOPP) & $11.6^{\mathrm{g}}$ & 1.4 & 7.6 & 1.6 & $p<.001$ \\
\hline Elision (CTOPP) & $10.7^{\mathrm{g}}$ & 1.3 & 7.4 & 3.6 & $p<.009$ \\
\hline Segmenting Nonwords (CTOPP) & $11.0^{\mathrm{g}}$ & 1.8 & 8.2 & 3 & $p<.016$ \\
\hline Phoneme Reversal (CTOPP) & $13.0^{\mathrm{g}}$ & 2.4 & 7.8 & 2.3 & $p<.001$ \\
\hline ACT no delay & $100.0^{\mathrm{e}}$ & 0 & 92.7 & 10.1 & $p<.027$ \\
\hline ACT 3-second delay & $85.5^{\mathrm{e}}$ & 12.9 & 65.5 & 18.1 & n.s. \\
\hline ACT 9-second delay & $69.1^{\mathrm{e}}$ & 28.8 & 47.3 & 24.1 & $p<.007$ \\
\hline ACT 18-second delay & $52.7^{\mathrm{e}}$ & 25.7 & 30.9 & 18.7 & $p<.034$ \\
\hline Rapid Letter Naming (CTOPP) & $10.4^{\mathrm{g}}$ & 2.8 & 9.8 & 3.4 & n.s. \\
\hline Rapid Color Naming (CTOPP) & $11.0^{\mathrm{g}}$ & 2.5 & 9.6 & 2.3 & n.s. \\
\hline
\end{tabular}

Note. n.s. $=$ not significant. WAIS-III $=$ Wechsler Adult Intelligence Scale $3^{\text {rd }}$ Ed. $($ Wechsler, 1997). WRMT-R $=$ Woodcock Reading Mastery Test-Revised (Woodcock, 1987). GORT-III = Gray Oral Reading Test $3^{\text {rd }}$ Ed. (Wiederholt \& Bryant, 1992). LAC Test (Lindamood, 1985). LAC-MAC = Lindamood Auditory Conceptualization-Multisyllable Auditory Conceptualization Test-Research Version. CTOPP $=$ Comprehensive Test of Phonological Processing (Wagner et al., 1999). ${ }^{a}$ WAIS-III Block Design and Vocabulary two-subtest estimated IQ. 'b(Oldfield, 1971). 'Socio-economic Status (Hollingshead, 1975); both groups scored in the medium business, minor professional, technical social strata classification, which may be high for most individuals diagnosed with dyslexia. ${ }^{\mathrm{d}}$ Weighted raw score. ${ }^{\mathrm{e} P e r c e n t}$ correct. ${ }^{\mathrm{f}}$ Standard scores with $M=100$ and $S D=15$. ${ }^{\mathrm{g}}$ Scaled scores with $M=10$ and $S D=3$. 
with dyslexia was withdrawn after a questionable history of Asperger's syndrome surfaced. Group matching was unaffected by participant removals. No participants reported a speech disorder, head injury, epilepsy, substance abuse, neurological or psychiatric disorder, visual or auditory acuity impairment, or more than two years of musical training. Participants were recruited by flyers, word of mouth, and printed announcements at two local dyslexia clinics. All participants who contacted the investigator agreed to participate in the study. Informed consent was obtained pursuant to a protocol approved by the University of Florida's Health Science Center Institutional Review Board.

\section{Paradigm Design}

During fMRI, participants maintained visual fixation on a cross viewed through a head coil mirror while pairs of pseudowords, pairs of tone series, or white noise were presented binaurally (Fig. 1). Blocks of stimuli were counterbalanced and pseudo randomly alternated with $12.5,15$, and 17.5 second blocks of white noise. Each stimuli block was fixed at 30 seconds by varying the response time after stimulus pairs from 2.4 to 3.7 seconds; response durations were derived from a pilot study with normal readers and adults diagnosed with dyslexia. Each run began with 17.5 seconds of silence, allowing adequate estimation of baseline fMRI signal, and lasted 4 minutes and 36 seconds. Participants completed four fMRI runs per stimuli type, totaling 12 blocks and 17 minutes and 44 seconds.

During pseudowords comparison, participants silently segmented each pseudoword into constituent phonemes and silently counted the total number of phonemes per pseudoword. Two pseudowords with the same number of phonemes $(2,3,4$, or 5$)$ were trained as target stimuli, indicated by a button press with the thumb of the dominant righthand. Two pseudowords with an unequal number of phonemes required no response.

In the tones comparison task, participants silently segmented and counted each tone in a series. Two series of tones with the same number of tones $(2,3,4$, or 5$)$ were

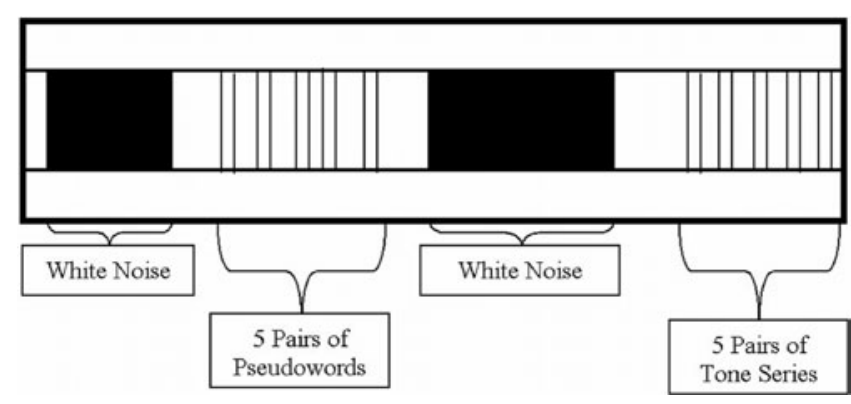

Fig. 1. Mixed block design with pseudowords, tones and white noise blocks pseudo randomized per run. trained as target stimuli. Participants responded to target stimuli by a button press with the thumb of the dominant hand. Two tone series with an unequal number of tones required no response.

Whereas sensorimotor, attention, and cognitive parameters were equal between the two tasks, the pseudowords comparison task required perception and segmentation of pseudowords instead of non-linguistic (pure tones) stimuli. Participants' button-press responses were recorded via observation of a light-box in the console room. The signal in voxels of cortical tissue, active during task performance, was expected to rise and fall with the alternation of task performance and white noise presentation. Because adults diagnosed with dyslexia show slower response rates to some auditory stimuli our paradigm utilized a block design that was insensitive to response rate and therefore it was not measured (Chait et al., 2007).

All pseudowords were monosyllable, digitally sampled at $44.1 \mathrm{kHz}$, normalized, and compressed to an equal amplitude (Cool Edit 2000, Syntrillium Software, 2000). A male speaker with Midwestern dialect of North American English produced all pseudowords with equivalent prosody. Monosyllable pseudowords contained two to five phonemes and followed English phonotactic conventions. Two pseudowords in a pair were presented binaurally and sequentially, separated by 1 second of silence. To prevent masking by white noise blocks, 250 milliseconds of silence preceded each pseudowords block. The average pseudoword frequency of occurrence value per run (Roberts, 1965) was equivalent across all runs. All four pseudowords comparison runs were equated on number of targets, number of targets per ordinal position, and on the average number of phonemes per pseudoword per run, 3.25 phonemes. Equating runs on average pseudoword frequency of occurrence values and average number of phonemes per run controlled for differential cortical activity because of variability in pseudoword novelty, complexity, or unequal word duration across the runs. For example, target pseudoword pairs with the same number of phonemes included /ravz/ \& /gluj/, /glo/ \& /igz/, /minz/ \& /mogz/, and /bo/ \& /ni/. Sample foil pairs, different number of phonemes per word, included /grom/\& /daj/, /uj/ \& /ezd/, /najd/ \& /ovz/, /lud/ \& /glabd/.

For tones comparison, each series included combinations of 500, 750, and $1000 \mathrm{~Hz}$ pure tones. Each tone was 200 milliseconds in duration, digitally sampled at $44.1 \mathrm{kHz}$, with equal maximum amplitude, and their amplitudes were equivalent to pseudoword stimuli. Tones in a series were separated by 250 milliseconds of silence and two series of tones comprised a pair. Five-pairs of tone series occurred per block (see Fig. 1). All four runs had equivalent number of targets, targets per ordinal position in the pairs within a block, tones per $\mathrm{Hz}$, and target pair combinations of tones. Also, 3.25 tones occurred on average per series across all four runs. To prevent masking by white noise blocks, tone comparison blocks were preceded by 250 milliseconds of silence. 


\section{Experimental Protocol}

Prior to fMRI, participants completed the State-Trait Anxiety Inventory (STAI) (Spielberger, 1983) to measure situational and typical anxiety levels. All participants received detailed instructions and practice on both fMRI tasks. During the pseudoword comparison task, phoneme counting was defined as counting only heard phonemes, with initial consonant blends, (e.g., bl, pl, tr...) and final consonant blends (e.g., nd, gd, bd. ..) considered as two phonemes. Unlimited practice occurred until participants demonstrated accurate task performance and reported confidence in their performance. During fMRI, task directions were reviewed prior to each run. At the end of a scanning session, each participant completed a debriefing questionnaire to assess strategy use during fMRI, measuring adherence to the fMRI task instructions.

Sufficient sound delivery of stimuli was ensured with audiological testing. Prior to participant scanning, an audiologist measured peak scanner noise (Bruel \& Kjer 2237 Controller) during an fMRI spiral sequence, 99.4 decibels. Binaural sound output from the MR compatible sound delivery system was verified with a measuring amplifier (Bruel \& Kjer type 2609) and an artificial ear (Bruel \& Kjer type 4152), 95 decibels. Foam ear inserts attenuated scanner noise by 30 decibels. Based on these measures, audiologist's professional opinion, and participants' post-scanning selfreport, the binaural sound delivery provided sufficient stimuli clarity. After the last participant's fMRI, repeat measurement of sound levels found all levels equivalent to initial measurements.

\section{fMRI Data Acquisition}

fMRI data were acquired using a two-shot spiral gradient echo sequence (Noll, Cohen, Meyer, \& Schneider, 1995) on a $3 \mathrm{~T}$ GE Signa LX scanner $(\mathrm{TE}=18 \mathrm{~ms}$; TR $=1250 \mathrm{~ms}$; $\mathrm{FA}=70^{\circ}, \mathrm{FOV}=20 \mathrm{~cm} ; 128 \times 128$ matrix, 24 contiguous sagittal $5.5 \mathrm{~mm}$ thick slices). The time required for acquisition of each whole-brain volume was $2500 \mathrm{~ms}$. A 124slice high-resolution T1 weighted 3D spoiled GRASS image set $\left(\mathrm{TE}=8 \mathrm{~ms}, \mathrm{TR}=23 \mathrm{~ms}\right.$, flip angle $=25^{\circ}$, slice thickness $=1.3 \mathrm{~mm}, \mathrm{FOV}=24 \mathrm{~cm}, 256 \times 256$, ) provided anatomic reference for functional data.

\section{fMRI Data Analysis}

For all functional runs, head motion detection and correction, image registration, spatial normalization and filtering, and conversion to a standard anatomic reference (Talairach \& Tournoux, 1998) were performed with the Analysis of Functional Neuroimages (AFNI) software (Cox, 1996). All fMRI runs were detrended of low frequency drifts and concatenated per task. Voxels with a standard deviation of signal change exceeding $5 \%$ of mean signal intensity were masked to remove artifacts caused by excessive motion, large vessel effects and other nuisance artifacts. Deconvo- lution analysis was used to estimate the impulse response function for each condition (Cox, 1996) with a maximum lag of 16 images (40 seconds) to allow return to baseline of hemodynamic response function (HRF). Area under the curve (AUC) for estimated HRF was calculated voxel by voxel for each task. AUC values quantified BOLD signal change for each block. Individual structural and functional images were resampled at $1 \mathrm{~mm}^{3}$ voxel-resolution, warped to standard Talairach space (Talairach \& Tournoux, 1998). The Talairach Method of Piecewise Linear Scaling (TMPLS), adapted in AFNI, was used to locate anatomical landmarks and perform the necessary rotation and linear scaling to transform anatomic and functional datasets into Talairach space. Images were smoothed with a $5 \mathrm{~mm}$ full-width halfmaximum isotropic Gaussian filter to compensate for interparticipant variability in structural and functional anatomy.

For within-group analyses, voxel-wise repeated measures $t$-tests were conducted, comparing each experimental task (normalized AUC) to baseline (white noise); AUC was divided by the mean intensity of the entire time series to produce a normalized AUC. For between-groups analyses (adults diagnosed with dyslexia $v s$. normal readers), voxelwise unpaired $t$-tests were conducted comparing the normalized AUC values between two groups across each stimulus type or a contrast of the two stimulus types (tones $v s$. pseudowords). To guard against errors in specificity, these group $t$-score statistical parametric maps were thresholded at $t \geq 3.58$ ( $p$-values of $\leq 0.005$ ) and clustered with connectivity distance $1.8 \mathrm{~mm}$ and minimum volume threshold of $150 \mathrm{~mL}$ (Forman et al., 1995). Neuroanatomical localization of clusters was based on clusters' 3-plane borders and voxel of maximum intensity coordinates.

\section{RESULTS}

\section{Behavioral Performance}

Reliable and greater than chance task accuracy during fMRI of both tones comparison and pseudowords comparison was present for normal readers and adults diagnosed with dyslexia. On the tones comparison task normal readers and adults diagnosed with dyslexia demonstrated a high percent of average correct responses $(M=99.1, S D=2.0, M=$ 91.4, $S D=17.3$, respectively); however, no significant differences in performance accuracy existed between the two groups $(p<.172)$. As expected, there was a greater frequency of response to target stimuli than foils $(p<.0001$ and $p<.0001$, respectively). On pseudowords comparison, normal readers and adults diagnosed with dyslexia had a low average percent correct $(M=64.6, S D=10.4 ; M=$ $56.8, S D=11.5$, respectively); however, no significant differences in performance accuracy existed between the two groups $(p<.158)$. As expected, both normal readers and adults diagnosed with dyslexia demonstrated a frequency of response to pseudoword targets that was significantly greater than to foils ( $p<.0003$ and $p<.01$, respectively). Participants' responses to post-scanning strategy debriefing 
Table 2. Normal readers' activity during tones comparison versus white noise

\begin{tabular}{lcllc}
\hline \hline Region & $\begin{array}{c}\text { Volume } \\
\mu \mathrm{L}^{\mathrm{a}}\end{array}$ & $\begin{array}{c}\text { Brodmann } \\
\text { area }\end{array}$ & $\begin{array}{c}\text { Local maximum } \\
(\mathrm{x}, \mathrm{y}, \mathrm{z})\end{array}$ & $\begin{array}{c}\text { Maximum } \\
t \text {-value }\end{array}$ \\
\hline L-TTG, Ins, STG, MTG & 2345 & $41,22,21$ & $(-43,-18,6)$ & $8.04^{*}$ \\
R-STG, TTG, MTG & 1930 & $22,41,42,21$ & $(52,-20,4)$ & $7.12^{*}$ \\
R-STG, MTG & 858 & $42,22,21$ & $(56,-22,14)$ & $7.17^{*}$ \\
R-SFG & 560 & 6 & $(3,7,57)$ & $5.36^{*}$ \\
L-TTG, STG, SMG & 321 & $41,22,42,40$ & $(-44,-34,17)$ & $5.51^{*}$ \\
R-STG & 228 & $22,38^{\mathrm{b}}$ & $(49,4,-5)$ & $5.41^{*}$ \\
\hline \hline
\end{tabular}

Note. $\mathrm{L}=$ left. $\mathrm{R}=$ right. $\mathrm{Ins}=$ insula. $\mathrm{MTG}=$ middle temporal gyrus. $\mathrm{SFG}=$ superior frontal gyrus. $\mathrm{SMG}=$ supramarginal gyrus. $\mathrm{STG}=$ superior temporal gyrus. TTG $=$ transverse temporal gyrus. Regions listed from largest to smallest cluster volume.

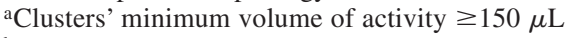

${ }^{b}$ Not visible in Figure 2 because of opacity of image.

$* p \leq .005$.

questionnaires indicated a consistent use of the trained auditory segmentation and counting strategy for both scanning tasks.

\section{Imaging Results}

Table 2 and Figure 2A (left panel) show distinct patterns of neural activity in normal readers during tones comparison versus baseline white noise. Normal readers showed bilateral activity in primary and secondary auditory cortex (BA $41,42,21,22)$. Left hemisphere activity occurred in SMG (BA 40). Right hemisphere activity appeared in temporal pole (BA 38; not visible on Fig. 2) and superior frontal gyrus (BA 6). Both participant groups showed bilateral frontal and posterior perisylvian activity (Fig. 2A and 2B left panel).

Table 3 and Figure 2A (right panel) show unique patterns of neural activity for normal readers during pseudowords comparison versus baseline white noise. Normal readers exhibited bilateral activity in primary and secondary auditory cortex (BA 41, 42, 21, 22) and pre-SMA. Right hemisphere activity occurred in anterior cingulate gyrus (BA 32) and middle frontal gyrus (BA 8). Decreased activity existed in left superior parietal lobe (BA 7) and post-central gyrus (BA 31). Both participant groups showed bilateral posterior perisylvian activity (Fig. 2A and 2B right panel).

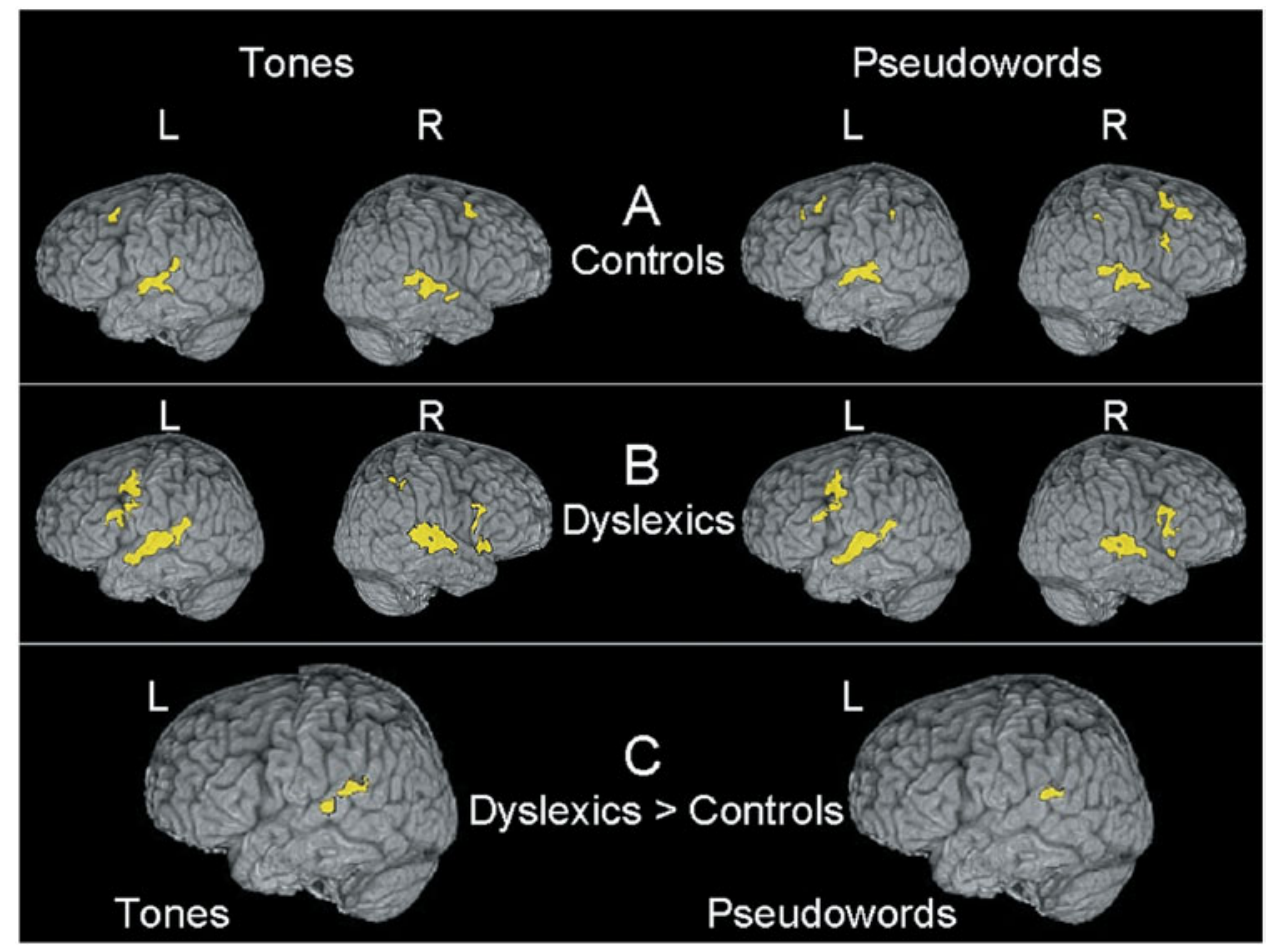

Fig. 2. Activity for normal readers (A), adults diagnosed with dyslexia (B) and significant between group differences (C) during auditory working memory for tones (left panel) and auditory working memory for pseudowords (right pane) 
Table 3. Normal readers' activity during pseudowords comparison versus white noise

\begin{tabular}{lcccr}
\hline \hline Region & $\begin{array}{c}\text { Volume } \\
\mu \mathrm{L}^{\mathrm{a}}\end{array}$ & $\begin{array}{c}\text { Brodmann } \\
\text { area }\end{array}$ & $\begin{array}{c}\text { Local maximum } \\
(\mathrm{x}, \mathrm{y}, \mathrm{z})\end{array}$ & $\begin{array}{c}\text { Maximum } \\
t \text {-value }\end{array}$ \\
\hline R-STG, TTG, MTG & 2605 & $22,41,42,21$ & $(49,-4,-4)$ & $7.17^{*}$ \\
L-STG, TTG, MTG & 2288 & $22,41,21,42$ & $(-62,-18,6)$ & $7.56^{*}$ \\
R-pre-SMA, MeFG, SFG & 1188 & $6,8,32$ & $(3,21,42)$ & $7.78^{*}$ \\
R-MFG & 328 & 8 & $(41,14,35)$ & $5.40^{*}$ \\
L-PoCG, L-MPL & 170 & 31,7 & $(-5,-46,42)$ & $-4.19^{*}$ \\
\hline \hline
\end{tabular}

Note $\mathrm{L}=$ left. $\mathrm{R}=$ right. $\mathrm{MeFG}=$ medial frontal gyrus. $\mathrm{MFG}=$ middle frontal gyrus. $\mathrm{MPL}=$ medial parietal lobe. $\mathrm{MTG}=$ middle temporal gyrus. $\mathrm{PoCG}=$ posterior cingulate gyrus. Pre-SMA = pre-supplementary motor area. $\mathrm{SFG}=$ superior frontal gyrus, $\mathrm{STG}=$ superior temporal gyrus. TTG $=$ transverse temporal gyrus. Regions listed from largest to smallest cluster volume. ${ }^{a}$ Clusters' minimum volume of activity $\geq 150 \mu \mathrm{L}$ $* p \leq .005$.

Table 4 shows results of contrasting pure tones (nonspeech) versus pseudowords (speech stimuli) tasks for normal readers. Two clusters demonstrated greater activity during tones comparison than pseudowords for normal readers. One cluster occurred in left STG (BA 22) and another in right cingulate gyrus (BA 32). No clusters showed greater activity for pseudowords than tones.

Table 5 shows differences between groups identified by comparing neural activity for adults diagnosed with dyslexia and normal readers during tones comparison, pseudowords comparison or a contrast of tones comparison versus pseudowords comparison. During tones comparison, two clusters in left STG and inferior parietal lobe (BA 22 and 40, $389 \mu \mathrm{L}$; BA 42, $349 \mu \mathrm{L}$ ) showed significantly more activity in adults diagnosed with dyslexia than normal readers (Fig. 2C, left panel). During pseudowords comparison, a cluster in left STG (BA 22, extending into BA 40, $295 \mu \mathrm{L}$ ) exhibited more activity in adults diagnosed with dyslexia than normal readers (Fig. 2C, right panel). Between groups differences on a tones versus pseudowords contrast revealed one cluster with greater activity in adults diagnosed with dyslexia than normal readers (left hemisphere STG, BA 42, tones comparison $>$ pseudowords comparison).

\section{DISCUSSION}

We used fMRI to identify neural activity associated with linguistic and nonlinguistic auditory working memory

Table 4. Normal readers' greater activity during tones versus pseudowords comparison

\begin{tabular}{lcccc}
\hline \hline Region & $\begin{array}{c}\text { Volume } \\
\mu \mathrm{L}^{\mathrm{a}}\end{array}$ & $\begin{array}{c}\text { Brodmann } \\
\text { area }\end{array}$ & $\begin{array}{c}\text { Local maximum } \\
(\mathrm{x}, \mathrm{y}, \mathrm{z})\end{array}$ & $\begin{array}{c}\text { Maximum } \\
t \text {-value }\end{array}$ \\
\hline L-STG & 216 & 22 & $(-49,-8,6)$ & $5.48^{*}$ \\
R-CG & 186 & 32 & $(10,39,0)$ & $5.44^{*}$ \\
\hline \hline
\end{tabular}

Note. $\mathrm{L}=$ left. $\mathrm{R}=$ right. $\mathrm{STG}=$ superior temporal gyrus. $\mathrm{CG}=$ cingulate gyrus. Regions listed from largest to smallest cluster volume.

${ }^{a}$ Clusters' minimum volume of activity $\geq 150 \mu \mathrm{L}$

$* p \leq .005$.
(AWM) comparison tasks in adults with normal reading and normal AWM skills versus adults with developmental phonological dyslexia and impaired AWM skills (per Auditory Consonant Trigrams Test (Stuss et al., 1988) and Lindamood Auditory Conceptualization Test (Lindamood, 1985) performances). For this study, the salient cognitive components needed for the AWM comparison task with pseudowords were speech perception, phonological awareness, AWM, comparison of phoneme sequences, and motoric response. To determine if adults diagnosed with dyslexia demonstrated a speech-specific deficit in AWM, we also measured neural activity during AWM of non-linguistic stimuli (pure tones). The salient cognitive components for the AWM comparison task for tones included auditory perception, AWM, comparison of tone sequences and motoric response. Therefore, the primary difference between tasks was the linguistic versus non-linguistic stimuli used for AWM.

Behavioral testing provided evidence of differential cognitive abilities between the adults diagnosed with dyslexia, and those with normal reading skills in the fMRI tasks' targeted cognitive areas of perception and explicit AWM comparison of linguistic stimuli. Test results indicated that compared to adults with normal reading skills, adults diagnosed with dyslexia were impaired in phonological awareness and AWM for linguistic stimuli. Additionally, AWM deficits were also evident during the complex linguistic analyses required by the LAC Test (Lindamood, 1985). However, despite these cognitive deficits in the adults diagnosed with dyslexia, the groups' performance accuracy was equivalent on both fMRI AWM tasks (tones and pseudowords comparisons); this implied that differences in neural activity were not likely caused by differences in task accuracy but may indicate underlying differences in the neural substrates of task performance.

During both fMRI AWM tasks, neural activity was observed in temporal, inferior parietal, and frontal regions for both groups. However, on between groups comparisons within each task the adults diagnosed with dyslexia showed greater activity than normal readers in the left auditory association cortex (BA 22) and supramarginal gyrus (BA 40). 
Table 5. Adults diagnosed with dyslexia exhibit greater activity than normal readers

\begin{tabular}{lccccc}
\hline \hline Comparison & Region & $\begin{array}{c}\text { Volume } \\
\mu \mathrm{L}^{\mathrm{a}}\end{array}$ & $\begin{array}{c}\text { Brodmann } \\
\text { area }\end{array}$ & $\begin{array}{c}\text { Local maximum } \\
(\mathrm{x}, \mathrm{y}, \mathrm{z})\end{array}$ & $\begin{array}{c}\text { Maximum } \\
t \text {-value }\end{array}$ \\
\hline Tones versus white noise & L-STG & 389 & 22,40 & $(-57,-39,20)$ & $4.84^{*}$ \\
& L-STG & 349 & 42 & $(-57,-26,10)$ & $5.65^{*}$ \\
Pseudowords versus white noise & L-STG & 295 & 22,40 & $(-58,-38,20)$ & $4.93^{*}$ \\
Tones versus Pseudowords & b-STG & 573 & 42 & $(-51,-29,10)$ & $5.48^{*}$ \\
\hline \hline
\end{tabular}

Note. $\mathrm{L}=$ left. $\mathrm{STG}=$ superior temporal gyrus.

aClusters' minimum volume of activity $\geq 150 \mu \mathrm{L}$

${ }^{\mathrm{b}}$ Cluster represents greater activity for tones than pseudowords. $* p \leq .005$.

As expected, differential activity in left primary auditory cortex (BA 42) occurred only during the tones comparison task, but again adults diagnosed with dyslexia exhibited more neural activity than normal readers in BA 42. To identify speech-specific regions of cortical activity, we subtracted the linguistic from non-linguistic activity within groups and then compared these differences between groups. Contrary to expectations of differential cortical activity with linguistic stimuli and similar cortical activity with nonlinguistic stimuli, the adults diagnosed with dyslexia showed greater activity than normal readers in left primary or secondary auditory cortex for linguistic and non-linguistic stimuli. Also seeming contrary to expectations is the lack of a significant left hemisphere lateralization for pseudowords comparison versus baseline white noise. However, recent theory indicates that the perception of linguistic stimuli may evidence at best a "weak left-hemisphere bias at this (phonological) level of processing" (Hickok \& Poeppel, 2007). As these authors' point out, this does not argue against the classical account of left hemisphere lateralization for linguistic perception, rather, it indicates that linguistic hemispheric lateralization may be less evident in some fMRI studies. Overall, during AWM of tones or pseudowords, the adults diagnosed with dyslexia demonstrated greater activity in the left STG and SMG than did the normal readers.

There are several possible explanations for why adults diagnosed with dyslexia in this study might show differential neural activity during AWM tasks when compared to normal readers. Behavioral testing prior to fMRI indicated impaired abilities in phonological awareness, AWM, and comparison of linguistic stimuli for the adults diagnosed with dyslexia. Based on these data and prior reports of relationships between behavioral abilities and functional brain activity, between groups differences in neural activity could be expected in STG (phonological perception), Inferior Parietal Lobe (phonological storage) and Inferior Frontal Lobe (comparison). However, the adults diagnosed with dyslexia only showed differential neural activity in left STG (with overlap into SMG). Because both groups show neural activity in the same region of interest (STG) and based on postscanning reports of consistent strategy use between the groups during fMRI, it is unlikely that differences in neural activity could be caused by differential cognitive strategies.
For example, the normal readers and readers diagnosed with dyslexia in Rumsey et al.'s (1992) study did not have equivalent task accuracy nor similar patterns of neural activity. This makes it possible that differential neural activity in that study could be because of participants' differential strategy use, a finding in another study too (Pekkola et al., 2006). Our findings of similar task accuracy and similar regions of neural activity, but different degrees of neural activity between adults diagnosed with dyslexia and normal readers are consistent with Chait et al.'s (2007) report of similar findings with an auditory perception task. However, despite similar levels of accuracy between the groups in our study, we cannot rule out that the adults diagnosed with dyslexia may have utilized greater cognitive effort than the normal readers to perform these AWM comparison tasks. Greater effort during fMRI has been shown to correspond with greater areas of neural activity (Poldrack, 2000; Price $\&$ Friston, 1999). Thus, although our data does not support conclusions of differential cognitive strategy or levels of performance as possible cause of our neural activity findings, we cannot rule out the possibility that greater cognitive effort by the adults diagnosed with dyslexia contributed to our findings of greater activity in left STG.

Other possible explanations of our findings of greater activity in left STG for readers diagnosed with dyslexia include deficient echoic memory, impaired sound sequence discrimination, impaired auditory feature analysis skills and priming by a dominant hand motor movement. It is possible that echoic memory played a role in the groups' differential neural activity, as echoic memory would affect AWM and it cannot be isolated in this study's tasks. Because the AWM tasks in this study required sound sequence discrimination, it is not likely that this contributed to our results, as the initial portion of the LAC Test measures sound sequencing without an AWM comparison and no differences existed between the groups on this portion of the LAC Test. Auditory feature analysis is a low-level analysis that would be common to tone and pseudoword AWM tasks, but the contributions of auditory feature analysis to the neural activity differences cannot be ruled out, because no tasks without memory were administered in this study. Similarly, because of white matter pathways between SMA and STG, we cannot rule out that the right-handed button press response 
may have influenced activity in left STG during both tasks. However, because button press preparation most likely produces activity in mesial pre-SMA and SMA and the motor task was identical for both AWM tasks, this influence should have been similar for both groups of participants and for both tasks. Thus, it is not likely that our results can be exclusively attributed to impaired sound sequence discrimination, or motor task priming, but auditory feature analysis and impaired echoic memory may have contributed to the differences in neural activity.

Differential neural activity may be related to aberrant neuroanatomic structures. Ramus (2004) proposed that genetically driven, focal, cortical anomalies in the left perisylvian cortex are the primary cause of phonological impairments in developmental dyslexia. These cortical anomalies seem to develop in utero, and contribute to morphological differences in perisylvian language cortex in adults with developmental dyslexia (Eckert, 2004; Galaburda, 1999; Galaburda et al., 2006). These structural anomalies affect functional cortico-cortical connections and may contribute to impaired phonological processing/reading skills. Aberrant fiber tracts in left posterior temporo-parietal cortices covary with children's and adults' reading skills, with higher fractional anisotropy and coherence indexes coinciding with better reading skill (Deutsch et al., 2005; Klingberg et al., 2000). Likewise, failures in neural pruning might produce overabundant synaptic connections in a cortical region, contributing to increased neural activity in this region (Haier et al., 1995). However, thicker myelin sheaths and larger axon diameters account for greater white matter volume in normal readers' left posterior STG, providing efficient neural conduction in the left STG (Golestani et al., 2002). Also, these cortical anomalies may be related to subcortical structural anomalies in humans (Galaburda, 1999), and a reciprocal impact between cortical and subcortical anomalies may influence AWM and BOLD response in a cortical region. Because post-mortem neuroanatomic analyses identify aberrant structures in the left perisylvian region of adults with developmental dyslexia (Galaburda et al., 2006; Galaburda et al., 1985), disordered localization of function is a possible contributor to our enhanced BOLD findings. Thus, future studies combining functional and structural neuroimaging are needed to elucidate the relationships between neuroanatomic structure, behavioral functions, and neural activity.

Overall, our findings are consistent with and extend previous studies indicating that phonological processing and AWM are altered in individuals with developmental dyslexia. Whereas this study's generalizability is limited by both groups exhibiting superior intellectual abilities and having greater than 12 years of education, the study's unique contribution is that activity differences in the left temporoparietal region between dyslexics and controls during AWM tasks are not unique to speech stimuli. Also, the differential neural activity was predominately in the left superior temporal gyrus. Our primary findings of higher levels of neural activity in posterior superior temporal and inferior parietal regions and impaired AWM skills with linguistic stimuli in adults diagnosed with dyslexia compliments behavioral studies showing AWM deficits in developmental dyslexia (Banai \& Ahissar, 2006; Berninger et al., 2006; Kibby et al., 2004). The fact that activity differences between groups existed only in primary and secondary auditory cortex for linguistic and nonlinguistic stimuli suggests that the atypical activity during AWM tasks in these adults with developmental dyslexia could be because of their poor auditory perception, AWM, or comparison skills rather than poor frontal executive functions, phonological awareness skills, or speech perception skills. This study cannot determine exactly which of these impaired skills or which combination of impaired skills are causing the greater neural activity during AWM tasks in adults diagnosed with dyslexia. Future research that combines functional and structural neuroimaging with behavioral performance is needed to determine the precise mechanisms that account for temporoparietal activity differences during AWM in developmental dyslexia.

\section{ACKNOWLEDGMENTS}

This work was supported by Associate Investigator B3480H (Conway) and Research Career Scientist B3470S (Crosson) awards from the Department of Veterans Affairs Rehabilitation Research and Development Service, the Donald D. Hammill Foundation (Conway), American Psychological Foundation (Conway), Fred J. Wellington Foundation (Conway), grants P50 DC 03888 and R01 DC 007387 from the National Institute on Deafness and Other Communication Disorders (Crosson), and grant HD30988 from the National Institute of Child Health and Human Development (Torgesen).

\section{REFERENCES}

Baddeley, A. (2003). Working memory and language: An overview. Journal of Communication Disorders, 36, 189-208.

Banai, K. \& Ahissar, M. (2006). Auditory processing deficits in dyslexia: Task or stimulus related? Cerebral Cortex, 16, 1718-1728.

Berninger, V.W., Abbott, R.D., Thomson, J., Wagner, R., Swanson, H.L., Wijsman, E.M., \& Raskind, W. (2006). Modeling phonological core deficits within a working memory architecture in children and adults with developmental dyslexia. Scientific Studies of Reading, 10, 165-198.

Binder, J.R., Frost, J.A., Hammeke, T.A., Bellgowan, P.S., Springer, J.A., Kaufman, J.N., \& Possing, E.T. (2000). Human temporal lobe activation by speech and nonspeech sounds. Cerebral Cortex, 10, 512-528.

Bruck, M. (1993). Word recognition and component phonological processing skills of adults with childhood diagnosis of dyslexia. Developmental Review, 13, 258-268.

Chait, M., Eden, G., Poeppel, D., Simon, J.Z., Hill, D.F., \& Flowers, D.L. (2007). Delayed detection of tonal targets in background noise in dyslexia. Brain Language, 102, 80-90.

Champod, A.S. \& Petrides, M. (2007). Dissociable roles of the posterior parietal and the prefrontal cortex in manipulation and 
monitoring processes. Proceedings of the National Academy Science USA, 104, 14837-14842.

Cox, R.W. (1996). AFNI: Software for analysis and visualization of functional magnetic resonance neuroimages. Computers and Biomedical Research, 29, 162-173.

Demonet, J.F., Chollet, F., Ramsay, S., Cardebat, D., Nespoulous, J.L., Wise, R., Rascol, A., \& Frackowiak, R. (1992). The anatomy of phonological and semantic processing in normal subjects. Brain, 115, 1753-1768.

Deutsch, G.K., Dougherty, R.F., Bammer, R., Siok, W.T., Gabrieli, J.D., \& Wandell, B. (2005). Children's reading performance is correlated with white matter structure measured by diffusion tensor imaging. Cortex, 41, 354-363.

Eckert, M. (2004). Neuroanatomical markers for dyslexia: A review of dyslexia structural imaging studies. Neuroscientist, 10, $362-371$.

Fiez, J.A., Raichle, M.E., Miezin, F.M., \& Petersen, S.E. (1995). PET studies of auditory and phonological processing: Effects of stimulus characteristics and task demands. Journal of Cognitive Neuroscience, 7, 357-375.

Fletcher, J.M. \& Shaywitz, S.E. (1994). Cognitive profiles of reading disability: Comparisons of discrepancy and low achievement. Journal of Educational Psychology, 86, 6-23.

Forman, S.D., Cohen, J.D., Fitzgerald, M., Eddy, W.F., Mintun, M.A., \& Noll, D.C. (1995). Improved assessment of significant activation in functional magnetic resonance imaging (fMRI): Use of a cluster-size threshold. Magnetic Resonance Medicine, 33, 636-647.

Galaburda, A.M. (1999). Developmental dyslexia: A multilevel syndrome. Dyslexia: An International Journal of Research and Practice, 5, 183-191.

Galaburda, A.M., LoTurco, J., Ramus, F., Fitch, R.H., \& Rosen, G.D. (2006). From genes to behavior in developmental dyslexia. Nature Neuroscience, 9, 1213-1217.

Galaburda, A.M., Sherman, G.F., Rosen, G.D., Aboitiz, F., \& Geschwind, N. (1985). Developmental dyslexia: Four consecutive patients with cortical anomalies. Annuals of Neurology, 18, 222-233.

Golestani, N., Paus, T., \& Zatorre, R.J. (2002). Anatomical correlates of learning novel speech sounds. Neuron, 35, 9971010.

Hagman, J.O., Wood, F., Buchsbaum, M.S., Tallal, P., Flowers, L., \& Katz, W. (1992). Cerebral brain metabolism in adult dyslexic subjects assessed with positron emission tomography during performance of an auditory task. Archives of Neurology, 49, 734-739.

Haier, R.J., Chueh, D., Touchette, P., \& Lott, I. (1995). Brain size and cerebral glucose metabolic rate in nonspecific mental retardation and Down syndrome. Intelligence, 20, 191-210.

Hickok, G. \& Poeppel, D. (2007). The cortical organization of speech processing. Nature Reviews Neuroscience, 8, 393-402.

Hollingshead, A.B. (1975). Four factor index of social status. Unpublished manuscript, Yale University at New Haven.

Kibby, M.Y., Marks, W., Morgan, S., \& Long, C.J. (2004). Specific impairment in developmental reading disabilities: A working memory approach. Journal of Learning Disabilities, 37, 349.

Klingberg, T., Hedehus, M., Temple, E., Salz, T., Gabrieli, J.D., Moseley, M.E., \& Poldrack, R.A. (2000). Microstructure of temporo-parietal white matter as a basis for reading ability: Evidence from diffusion tensor magnetic resonance imaging. Neuron, 25, 493-500.
Liebenthal, E., Binder, J.R., Spitzer, S.M., Possing, E.T., \& Medler, D.A. (2005). Neural substrates of phonemic perception. Cerebral Cortex, 15, 1621-1631.

Lindamood, C.H. (1985). The LAC Test (Rev. ed.). Allen, TX: DLM Teaching Resources.

Lyon, G.R., Shaywitz, S.E., \& Shaywitz, B.A. (2003). A definition of dyslexia. Annals of Dyslexia, 53, 1-14.

Noll, D.C., Cohen, J.D., Meyer, C.H., \& Schneider, W. (1995). Spiral K-space MR imaging of cortical activation. Journal of Magnetic Resonance Imaging, 5, 49-56.

Oldfield, R.C. (1971). The assessment and analysis of handedness: The Edinburgh Inventory. Neuropsychologia, 9, 97-113.

Pekkola, J., Laasonen, M., Ojanen, V., Autti, T., Jaaskelainen, I.P., Kujala, T., \& Sams, M. (2006). Perception of matching and conflicting audiovisual speech in dyslexic and fluent readers: An fMRI study at 3 T. Neuroimage, 29, 797-807.

Poldrack, R.A. (2000). Imaging brain plasticity: Conceptual and methodological issues - a theoretical review. Neuroimage, 12, $1-13$.

Price, C.J. \& Friston, K.J. (1999). Scanning patients with tasks they can perform. Human Brain Mapping, 8, 102-108.

Ramus, F. (2004). Neurobiology of dyslexia: A reinterpretation of the data. Trends in Neuroscience, 27, 720-726.

Roberts, A.H. (1965). A statistical linguistic analysis of American English. The Hague: Mouton.

Rumsey, J.M., Andreason, P., Zametkin, A.J., Aquino, T., King, A.C., Hamburger, S.D., Pikus, A., Rapoport, J.L., \& Cohen, R.M. (1992). Failure to activate the left temporoparietal cortex in dyslexia. An oxygen 15 positron emission tomographic study. Archives of Neurology, 49, 527-534.

Shaywitz, S.E., Morris, R., \& Shaywitz, B.A. (2008). The education of dyslexic children from childhood to young adulthood. Annual Review of Psychology, 59, 451-475.

Spielberger, C.D. (1983). Manual for the State-Trait Anxiety Inventory. Palo Alto, CA: Consulting Psychologists Press, Inc.

Stanovich, K.E. \& Siegel, L.S. (1994). Phenotypic performance profile of children with reading disabilities: A regressionbased test of. Journal of Educational Psychology, 86, 24-53.

Stuss, D.T., Stethem, L.L., \& Pelchat, G. (1988). Three tests of attention and rapid information processing: An extension. Clinical Neuropsychologist, 2, 246-250.

Swanson, H.L. \& Siegel, L. (2001). Learning disabilities as a working memory deficit. Issues in Education, 7, 1.

Talairach, J. \& Tournoux, P. (1998). Co-Planar Stereotaxic Atlas of the Human Brain. New York: Thieme Medical Publishers.

Tallal, P. (1980). Auditory temporal perception, phonics, and reading disabilities in children. Brain Language, 9, 182-198.

Torgesen, J.K. (1985). Memory Processes in Reading Disabled Children. Journal of Learning Disabilities, 18, 350-357.

Wager, T.D. \& Smith, E.E. (2003). Neuroimaging studies of working memory: A meta-analysis. Cognitive, Affective \& Behavioral Neuroscience, 3, 255-274.

Wagner, R.K., Torgesen, J.K., \& Rashotte, C.A. (1999). CTOPP, Comprehensive Test of Phonological Processing. Austin, TX: PRO-ED.

Wechsler, D. (1997). Wechsler Adult Intelligence Scale-Third Edition. San Antonio, TX: The Psychological Corporation.

Wiederholt, J.L. \& Bryant, B.R. (1992). Gray Oral Reading Test: Third Edition. Austin, TX: Pro-Ed, Inc.

Wolf, M. \& Bowers, P.G. (1999). The double-deficit hypothesis for the developmental dyslexias. Journal of Educational Psychology, 91, 415-438. 
Woodcock, R.W. (1987). Woodcock Reading Mastery TestsRevised. Circle Pines, MN: American Guidance Service, Inc.

Zatorre, R.J., Evans, A.C., Meyer, E., \& Gjedde, A. (1992). Lateralization of phonetic and pitch discrimination in speech processing. Science, 256, 846-849.
Zatorre, R.J., Meyer, E., Gjedde, A., \& Evans, A.C. (1996). PET studies of phonetic processing of speech: Review, replication, and reanalysis. Cerebral Cortex, 6, 21-30. 